\title{
Pharmacological effects of a novel isosorbide-based butyrylcholinesterase inhibitor
}

D. Khan, J.F. Gilmer, C.G. Carolan, J.M. Gaynor, S.A. Ryder

School of Pharmacy and Pharmaceutical Sciences, Trinity College Dublin, Dublin 2, Ireland

E-mail: sryder@tcd.ie (S.A. Ryder)

NOTICE: this is the author's version of a work that was accepted for publication in Chemico-Biological Interactions following peer review. Changes resulting from the publishing process, such as peer review, editing, corrections, structural formatting, and other quality control mechanisms may not be reflected in this document. Changes may have been made to this work since it was submitted for publication. A definitive version was subsequently published in Chemico-Biological Interactions, [Vol. 175, Issues 1-3 (Sep 2008)], DOI: 10.1016/j.cbi.2008.05.024.

Direct links to the definitive publisher-authenticated version [Khan D, Gilmer JF, Carolan CG, Gaynor JM, Ryder SA. Pharmacological effects of a novel isosorbide-based butyrylcholinesterase inhibitor. Chem Biol Interact. 2008 Sep 25;

175(1-3):231-4]:

http://www.sciencedirect.com/science/article/pii/S000927970800286X

http://dx.doi.org/10.1016/j.cbi.2008.05.024

\section{Abstract}

Isosorbide-2-benzylcarbamate-5-benzoate, a novel butyrylcholinesterase inhibitor, shows interspecies variation in its inhibitory activity ( $\left(\mathrm{C}_{50}\right.$ of $4.3 \mathrm{nM}$ for human plasma butyrylcholinesterase, but $1.09 \mu \mathrm{M}$ for mouse plasma butyrylcholinesterase). Stability studies revealed that this drug is resistant to hydrolysis by human plasma (no degradation in $1 \mathrm{~h})$. However, it was found to undergo rapid degradation when incubated with mouse plasma or mouse liver homogenate, yielding benzyl carbamate and benzoic acid. The addition of the carboxylesterase inhibitor bis-(4-nitrophenyl) phosphate (BNPP) inhibited the degradation of the novel drug, indicating that it may be a substrate for both butyrylcholinesterase and carboxylesterase. The absence of carboxylesterase from human plasma explains the drug's stability in this medium. In vivo, pharmacodynamic studies on single doses of $1 \mathrm{mg} / \mathrm{kg}$ to naïve male C57BL/6 mice revealed maximal plasma butyrylcholinesterase inhibition $20 \mathrm{~min}$ after intraperitoneal administration ( 60\% inhibition) and $1 \mathrm{~h}$ after administration by gavage ( $45 \%$ inhibition). While this plasma butyrylcholinesterase inhibition was short-lived, the drug also penetrated the blood-brain barrier resulting in a slight (10-15\%) but persistent ( $\geq 72 \mathrm{~h})$ reduction in brain butyrylcholinesterase activity.

Keywords Butyrylcholinesterase $\bullet$ Inhibition $\bullet$ Carboxylesterase $\bullet$ Hydrolysis

\section{Introduction}

Butyrylcholinesterase (EC 3.1.1.8, BuChE) is found in neurons and glial cells in the human brain, and peripherally in serum [1]. Although the exact function of BuChE remains unclear, it is responsible for the metabolism of various drugs including the anaesthetic succinylcholine [2], and it efficiently hydrolyses acetylcholine (ACh) at high concentrations [3]. In Alzheimer's disease, BuChE may be increased $40-90 \%$ in the brain and high levels of BuChE are found in neuronal plaques $[4,5]$. Novel and potent inhibitors displaying exceptionally high selectivity for BuChE have been developed in our laboratories, providing a unique pharmacological opportunity to explore the physiological and pathological roles of BuChE and the potential for BuChE inhibition as a therapeutic strategy in Alzheimer's disease [6]. One such compound, isosorbide2-benzylcarbamate-5-benzoate (compound 51, Fig. 1), which displays $>60,000$-fold selectivity for BuChE over acetylcholinesterase $(\mathrm{AChE})$, has been tested in vitro and in vivo. This paper describes studies intended to reconcile the metabolism of compound 51 with its in vivo activity.<smiles>O=C(Nc1ccccc1)O[C@H]1CO[C@@H]2[C@@H](OC(=O)c3ccccc3)CO[C@H]12</smiles>

Fig. 1. Compound 51, isosorbide-2-benzylcarbamate-5-benzoate. 


\section{Methods}

\subsection{In vitro butyrylcholinesterase inhibition}

BuChE inhibition was measured in vitro using a modification of the Ellman assay [7] employing butyrylthiocholine as substrate [8] and a standardized BuChE concentration $\left(5.88 \mu \mathrm{mol} \mathrm{min} \mathrm{m}^{-1} \mathrm{ml}^{-1}\right)$. Plasma was removed from fresh blood centrifuged at $3000 \mathrm{rpm}$ for $10 \mathrm{~min}$ at $4^{\circ} \mathrm{C}$ (Sorvall RT6000B). A stock solution of the drug was prepared in a 1:3 solution of acetonitrile:PBS (phosphate buffered saline) pH 8.0 from which serial dilutions (100 $\mu \mathrm{M}$ to $1 \mathrm{nM}$ ) were made. The drug and plasma were incubated in $0.1 \mathrm{M} \mathrm{PBS}\left(\mathrm{pH}\right.$ 8.0) with $0.3 \mathrm{mM}$ DTNB (5-5'-dithiobis(2-nitrobenzoic) acid) at $37^{\circ} \mathrm{C}$ for $30 \mathrm{~min}$. Thereafter $0.5 \mathrm{mM}$ of $\mathrm{BTCl}$ (butyrylthiocholine iodide) was added and the absorbance was measured at $405 \mathrm{~nm}$. IC 50 values were determined from non-linear regression plots using Graph-Pad Prism 4.0.

\subsection{In vivo butyrylcholinesterase inhibition}

Healthy naïve male C57BL/6 mice (Harlan, UK) were housed singly with ad libitum access to food and water under a $12 \mathrm{~h}$ light/dark cycle. A single dose of compound $51(1 \mathrm{mg} / \mathrm{kg}$ ) was administered intraperitoneally (ip) or orally (po). At various time points ( $n=3$ per timepoint) submandibular blood samples were collected in lithium heparinized Microvette tubes and centrifuged at $3000 \mathrm{rpm}$ for $10 \mathrm{~min}$ at $4^{\circ} \mathrm{C}$. Plasma BuChE activity was determined using the Ellman assay [7] as previously described. Absorbance values were plotted using GraphPad Prism 4.0.

At the study endpoints, 24,48 and $72 \mathrm{~h}$ post administration of the compound, the level of BuChE inhibition in the brain was assessed. The animals were euthanized by carbon dioxide asphyxiation and the brains were carefully removed and placed on ice until processed. The brain samples were homogenized in physiological saline on ice in three 5-s bursts using an Ultraturrax T10 (IKA-Werke). The homogenate was centrifuged at 10,000 rpm for $10 \mathrm{~min}$ at $4^{\circ} \mathrm{C}$. BuChE activity was analysed using the Ellman assay [7].

\subsection{Hydrolysis studies}

The rate of hydrolysis of compound 51 was determined in vitro in human plasma, mouse plasma and mouse liver homogenate using a gradient HPLC method.

\subsubsection{Preparation of plasma samples}

Fresh plasma samples were prepared by centrifugation of heparinized venous human or mouse blood for $10 \mathrm{~min}$ at 3000 $\mathrm{rpm}$ at $4^{\circ} \mathrm{C}$. One millilitre of plasma was added to $2 \mathrm{ml}$ of PBS pH 7.4 (33\% solution).

\subsubsection{Preparation of liver samples}

Mouse liver was homogenized on ice in 10 volumes of PBS pH 7.4 in three 5-s bursts using an Ultra-turrax T-10. The homogenate was then centrifuged for $10 \mathrm{~min}$ at $10,000 \mathrm{rpm}$ and $4^{\circ} \mathrm{C}$. Liver homogenate $(20 \mu \mathrm{l})$ was added to $3 \mathrm{ml}$ of PBS pH 7.4.

\subsubsection{Hydrolysis}

One hundred microlitres of compound $51(8 \mu \mathrm{M})$ in acetonitrile was added to the plasma/buffer or liver/buffer solution and incubated at $37^{\circ} \mathrm{C}$. Aliquots $(250 \mu \mathrm{l})$ were taken at various time points and transferred to Eppendorfs containing $500 \mu \mathrm{l}$ of a $2 \%$ zinc sulfate solution in acetonitrile (1:1). The Eppendorfs were then centrifuged at 10,000 rpm for $7 \mathrm{~min}$. A $20 \mu \mathrm{l}$ aliquot of the clear supernatant was analysed by HPLC.

Hydrolysis studies using the carboxylesterase inhibitor, bis-(4-nitrophenyl) phosphate (BNPP) were also carried out to determine if carboxylesterase played a role in the hydrolysis of compound 51 . BNPP $(100 \mu \mathrm{M})$ was incubated at $37^{\circ} \mathrm{C}$ with the mouse plasma or liver homogenate for 10 min prior to drug addition.

\subsubsection{Chromatography}

High-performance liquid chromatography was performed using a system consisting of a Waters 600 pump and controller, Waters 717 autosampler and a Waters 2996 photodiode-array (PDA) detector controlled by Empower 2 software (Waters). A Hichrom Nucleosil C18 $4.0 \mathrm{~mm} \times 250 \mathrm{~mm}$ column was used. The plasma and liver samples were both analysed using a gradient method consisting of a $16 \mathrm{mM}$ phosphate buffer $(\mathrm{pH} \mathrm{2.5)}$ : acetonitrile 80:20 for the first 10 min, grading to 20:80 for the next $5 \mathrm{~min}$, then 60:40 for the next $2 \mathrm{~min}$ and finally returning to 80:20 for the last $3 \mathrm{~min}$ of the $20 \mathrm{~min}$ run. The flow rate was $1 \mathrm{ml} / \mathrm{min}$ and the buffer and acetonitrile were helium sparged at a rate of $20 \mathrm{ml} / \mathrm{min}$. The retention times in this system were $9.5 \mathrm{~min}$ for benzyl carbamate, $10.0 \mathrm{~min}$ for benzoic acid and $15.2 \mathrm{~min}$ for compound 51 . Peak areas were compared quantitatively with external standards of approximately the same concentration. The gradient method was validated for specificity, sensitivity and precision.

\subsection{Statistical analysis}

BuChE activity data were entered into SPSS v.14 (SPSS Inc., Chicago) for analysis. Standard descriptive parameters were calculated and significant differences were determined using Student's $t$-test where appropriate, taking $p<0.05$ to be significant. 


\section{Results and discussion}

Compound 51 (isosorbide-2-benzylcarbamate-5-benzoate) was synthesised in house as part of an ongoing programme of research into isosorbide-based cholinesterase inhibitors, and has proved to be a potent inhibitor of human plasma BuChE in vitro. However, interspecies differences were apparent, its $I C_{50}$ of $4.3 \mathrm{nM}$ in human plasma contrasting with an $I C_{50}$ of $1.09 \mu \mathrm{M}$ in mouse plasma.

In vivo, a single dose of $1 \mathrm{mg} / \mathrm{kg}$ given to male C57BL/6 mice displayed maximal plasma BuChE inhibition 20 min after ip administration ( $60 \%$ inhibition), and $1 \mathrm{~h}$ after administration po ( $45 \%$ inhibition). However, the duration of action was short-lived by both routes, restoration of BuChE activity being substantially complete within $24 \mathrm{~h}$ (Fig. 2). There was a subsequent increase in plasma BuChE activity $24 \mathrm{~h}$ after ip administration with a concomitant rise in liver production of the enzyme to $115 \%$. Combined with the fact that repeated dosing $(5 \mathrm{mg} / \mathrm{kg}$ or $10 \mathrm{mg} / \mathrm{kg}$ po daily) over a 1 -week period showed an increase in plasma BuChE activity, this suggests the existence of an efficient BuChE homeostatic mechanism.

Single doses of $1 \mathrm{mg} / \mathrm{kg}$ of compound 51 by the intraperitoneal and oral routes also caused reductions of 20 and $15 \%$, respectively in BuChE activity in the brain, indicating the ability of the drug to cross the blood-brain barrier. Furthermore, these reductions persisted for at least $72 \mathrm{~h}$ after dosing (Fig. 3). The superior BuChE inhibition achieved by this compound in human plasma, compared with its effects in mice, prompted exploration of the possibility that it is subject to more rapid metabolism in mice.

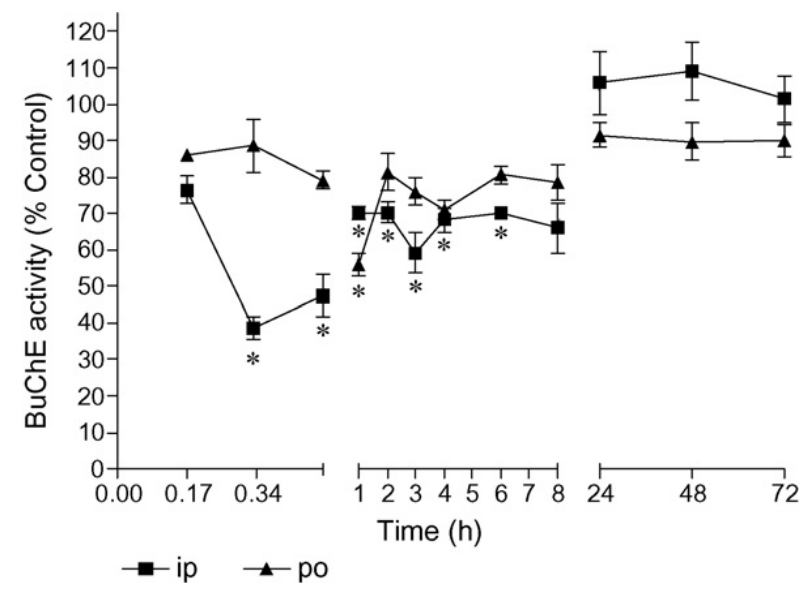

Fig. 2. Mouse plasma BuChE activity following a single intraperitoneal or oral dose $(1 \mathrm{mg} / \mathrm{kg}$ ) of compound 51. (Mean \pm standard deviation; $n=3$.) Asterisks indicate a significant difference in BuChE activity relative to controls $(p<0.05$, $t$-test).

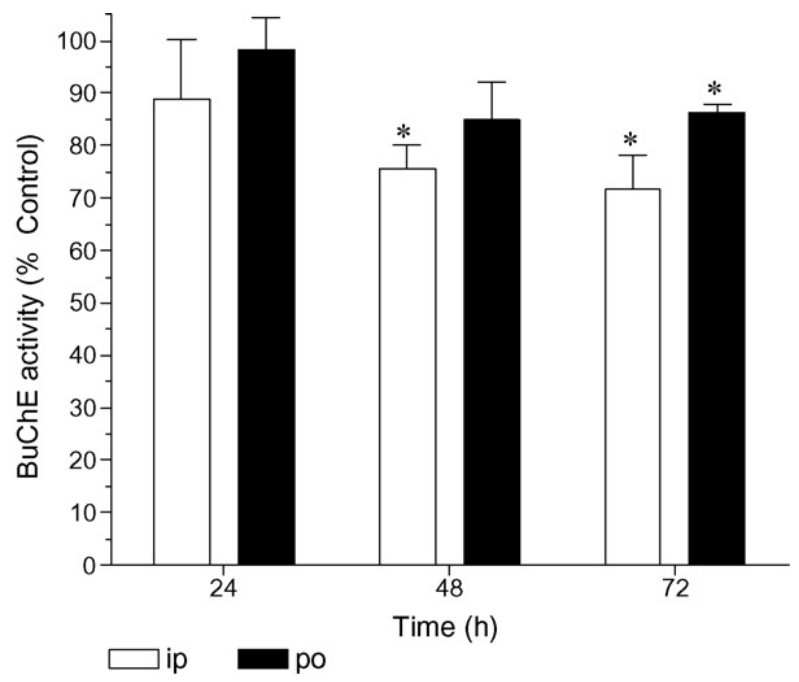

Fig. 3. Mouse brain BuChE activity following a single intraperitoneal or oral dose $(1 \mathrm{mg} / \mathrm{kg}$ ) of compound 51 . (Mean \pm standard deviation; $n=3$.) Asterisks indicate a significant difference in butyrylcholinesterase activity relative to controls $(p<0.05, t$-test).

Hydrolysis studies revealed that compound 51 is stable in human plasma: no decrease in drug concentration was seen over a 60-min period. In contrast, rapid hydrolysis was observed in mouse plasma and mouse liver. The concentration of the drug decreased over a period of 20 min with the concomitant formation of the metabolites benzyl carbamate and benzoic acid (Fig. 4).

Human plasma contains almost twice the concentration of BuChE in mouse plasma: 5 and 2.6 mg/l, respectively [9]. Accordingly, if BuChE were responsible for the hydrolysis of compound 51, its disappearance would have been more rapid in human than mouse plasma samples. The instability of compound 51 in mouse plasma and liver, allied with the higher $\mathrm{IC}_{50}$ observed in mouse plasma compared to the $\mathrm{IC}_{50}$ for human plasma BuChE, suggests that this drug is not a substrate of BuChE alone.

Human and mouse plasma differ insofar as mouse plasma contains an additional enzyme, carboxylesterase $[9,10](E C$ 3.1.1.1). Despite an abundance of carboxylesterase in human liver and small intestine, none is found in human plasma $[9,11]$. The hydrolysis of compound 51 by carboxylesterase was confirmed by incubation of the carboxylesterase inhibitor BNPP with mouse plasma and liver prior to addition of the drug. The inclusion of BNPP decreased the rate of degradation: in both mouse plasma and liver, compound 51 was hydrolysed over a period of $1 \mathrm{~h}$, compared with 20 min in the absence of BNPP (Fig. 5). This suggests that compound 51 is a substrate for both BuChE and carboxylesterase. 


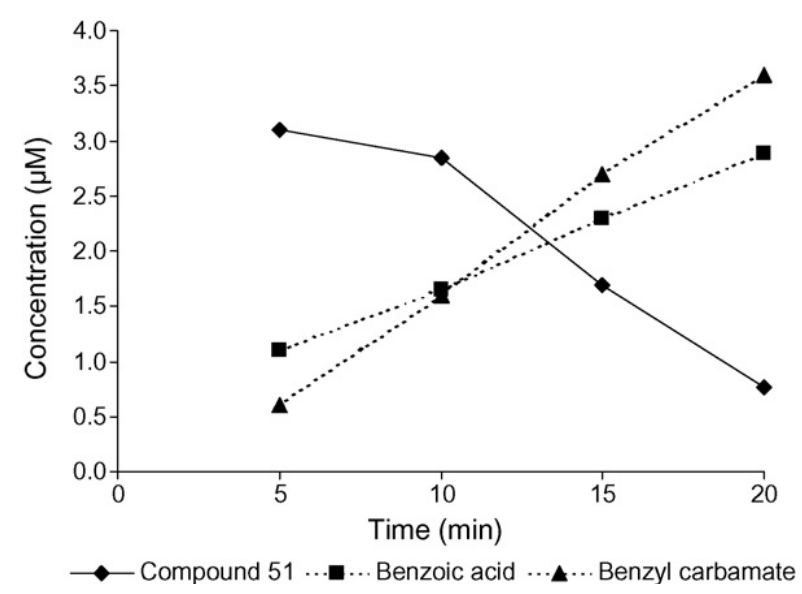

Fig. 4. Hydrolysis of compound 51 in a $33 \%$ mouse plasma solution incubated at $37^{\circ} \mathrm{C}(\mathrm{pH} 7.4)$.

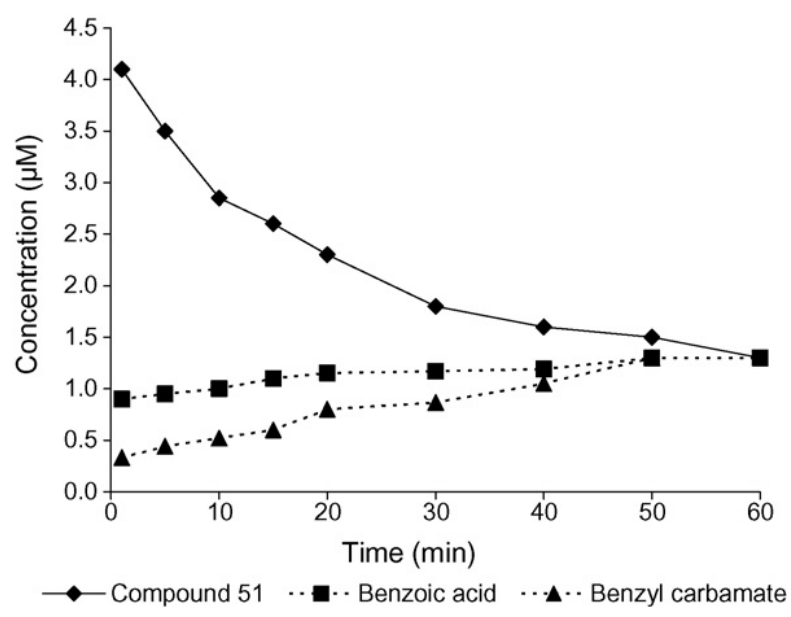

Fig. 5. Hydrolysis of compound 51 in a $33 \%$ mouse plasma solution incubated with $100 \mu \mathrm{M} \mathrm{BNPP}$ at $37^{\circ} \mathrm{C}(\mathrm{pH} 7.4)$.

\section{Conclusions}

Isosorbide-2-benzylcarbamate-5-benzoate (compound 51) is a potent inhibitor of human plasma BuChE but displays poorer inhibition of mouse plasma BuChE. This drug is stable in human plasma; however, it is rapidly lost from mouse plasma, explicable by its hydrolysis to benzyl carbamate and benzoic acid under the influence of carboxylase.

Pharmacodynamic studies show decreased plasma and brain BuChE activity following a single intraperitoneal or oral dose of $1 \mathrm{mg} / \mathrm{kg}$. The ability of this novel compound to cross the blood-brain barrier and reduce brain BuChE activity will prove interesting in cognitive tests in vivo, as BuChE has gained recognition as a new therapeutic target in Alzheimer's disease (AD). In the healthy brain, AChE is the predominant cholinesterase, with BuChE playing a supporting role. However, AChE levels show a pronounced decline at an early stage of $A D$ whereas BuChE levels increase as the disease progresses [12]. It has been proposed that BuChE may play a key part in compensating for AChE loss [13], limiting the value of selective AChE inhibition as a strategy for cholinergic enhancement in AD. While AChE inhibitors such as donepezil and galantamine have gained a place in the management of $A D$, the addition of a BuChE inhibitor such as compound 51 could enhance clinical efficacy.

\section{Acknowledgement}

This work was funded by Science Foundation Ireland (Research Frontiers Programme).

\section{References}

[1] E. Giacobini, Butryrlcholinesterase: Its Function and Inhibitors, Taylor \& Francis Ltd., London, 2003.

[2] S. Darvesh, D.A. Hopkins, C. Geula, Neurobiology of butyrylcholinesterase, Nat. Rev. Neurosci. 4 (2003) 131-138. PMID: 12563284. DOI: 10.1038/nrn1035.

[3] A. Silver, The Biology of Cholinesterases, Elsevier, Amsterdam, 1974.

[4] E.K. Perry, B.E. Tomlinson, G. Blessed, K. Bergmann, P.H. Gibson, R.H. Perry, Correlation of cholinergic abnormalities with senile plaques and mental test scores in senile dementia, Brit. Med. J. 2 (1978) 1457-1459. PMID: 719462. DOI: 10.1136/bmj.2.6150.1457.

[5] M.M. Mesulam, C. Geula, Butyrylcholinesterase reactivity differentiates the amyloid plaques of aging from those of dementia, Ann. Neurol. 36 (1994) 722-727. PMID: 7979218. DOI: 10.1002/ana.410360506.

[6] J.F. Gilmer, M.N. Lally, P. Gardiner, G. Dillon, J.M. Gaynor, S. Reidy, Novel isosorbide-based substrates for human butyrylcholinesterase, Chem. Biol. Interact. 157/158 (2005) 317-319. PMID: 16266694. DOI: 10.1016/j.cbi.2005.10.095.

[7] G.L. Ellman, K.D. Courtney, V. Andres Jr., R.M. Featherstone, A new and rapid colorimetric determination of acetylcholinesterase activity, Biochem. Pharmacol. 7 (1961) 88-95. PMID: 13726518. DOI: 10.1016/0006-2952(61)90145-9.

[8] A. Chatonnet, O. Lockridge, Comparison of butyrylcholinesterase and acetylcholinesterase, Biochem. J. 260 (1989) 625-634. PMID: 2669736.

[9] B. Li, M. Sedlacek, I. Manoharan, R. Boopathy, E.G. Duysen, P. Masson, O. Lockridge, Butyrylcholinesterase, paraoxonase, and albumin esterase, but not carboxylesterase, are present in human plasma, Biochem. Pharmacol. 70 (2005) 1673-1684. PMID: 16213467. DOI: 10.1016/j.bcp.2005.09.002.

[10] T. Satoh, M. Hosokawa, Structure, function and regulation of carboxylesterases, Chem. Biol. Interact. 162 (2006) 195-211. PMID: 16919614. DOI: 10.1016/j.cbi.2006.07.001.

[11] M. Taketani, M. Shii, K. Ohura, S. Ninomiya, T. Imai, Carboxylesterase in the liver and small intestine of experimental animals and human, Life Sci. 81 (2007) 924-932. PMID: 17764701. DOI: 10.1016/j.Ifs.2007.07.026.

[12] E. Giacobini, Cholinergic function and Alzheimer's disease, Int. J. Geriatr. Psychiatr. 18 (Suppl. 1) (2003) S1-S5. PMID: 12973744. DOI: 10.1002/gps.935.

[13] N.H. Greig, T. Utsuki, D.K. Ingram, Y. Wang, G. Pepeu, C. Scali, Q.S. Yu, J. Mamczarz, H.W. Holloway, T. Giordano, D. Chen, K. Furukawa, K. Sambamurti, A. Brossi, D.K. Lahiri, Selective butyrylcholinesterase inhibition elevates brain acetylcholine, augments learning and lowers Alzheimer beta-amyloid peptide in rodent, Proc. Natl. Acad. Sci. U.S.A. 102 (2005) 17213-17218. PMID: 16275899. DOI: 10.1073/pnas.0508575102. 